\title{
COMMENTS ON ITERATIVE SCHEMES FOR HIGH ORDER COMPACT DISCRETIZATIONS TO THE EXTERIOR HELMHOLTZ EQUATION
}

\author{
ELI TURKEL ${ }^{1}$
}

\begin{abstract}
We consider various formulations of higher order absorbing boundary conditions for the Helmholtz equation.
\end{abstract}

Mathematics Subject Classification. 65N06.

Received June 9, 2013. Revised July 1st, 2014

Published online January 16, 2015.

In [1] we considered the Helmholtz equation

$$
u_{x x}+u_{y y}+u_{z z}+k^{2} u=0
$$

with a simplified artificial absorbing boundary condition along $x=1$ of the form

$$
\frac{\partial u}{\partial x}+i \beta u=0 .
$$

We approximated this boundary condition by expanding $u$ in the $x$ direction to get

$$
\frac{\partial u}{\partial x}=\frac{u_{N+1}-u_{N-1}}{2 h}-\frac{h^{2}}{6} \frac{\partial^{3} u}{\partial x^{3}}-\frac{h^{4}}{120} \frac{\partial^{5} u}{\partial x^{5}}+O\left(h^{6}\right)
$$

where the $N+1$ th grid point lies outside the computational domain, and hence is a virtual point. The high order derivatives were found by differentiating (2) in the $x$ direction. After some manipulations this yields

$$
0=\frac{\partial u}{\partial x}+i k u=\frac{u_{N+1}+2 i \beta h\left(1-\frac{\beta^{2} h^{2}}{6}+\frac{\beta^{4} h^{4}}{120}\right) u_{N}-u_{N-1}}{2 h}+O\left(h^{6}\right) .
$$

However, in general given a boundary condition at $x=1$ it is not legitimate to differentiate (2) in the direction $x$ but only in perpendicular directions. The calculations in [1] showed fourth order accuracy only because the solution chosen had the form

$$
u(x, y)=\mathrm{e}^{i \beta x} f(y)
$$

and so satisfies (2) exactly. Hence, the numerical absorbing boundary condition (3) is correct only if the solution behaves like (4), at least to fourth order accuracy, in the neighborhood of the absorbing boundary, $x=1$.

Keywords and phrases. Helmholtz equation, absorbing boundary conditions.

1 School of Mathematical Sciences, Tel Aviv University, Ramat Aviv, 69978 Tel Aviv, Israel. eliturkel@gmail.com 
A different approach, which does not suffer from this difficulty, is to consider a Neumann boundary condition $u=g(y, z)$ at $x=1$ which is constructed by using the Helmholtz equation to reduce high order derivatives to lower order derivatives. In [2] we constructed a compact sixth order approximation to a Neumann boundary condition for the Helmholtz equation even with a variable wavenumber $k$. Let $\delta_{x}$ and $\delta_{x x}$ be the standard central first and second differences in the $x$ direction. Then this was given by

$$
\begin{aligned}
\delta_{x} u & +\frac{h^{2}}{6}\left(k^{2}\right)_{x} u-\frac{h^{4}}{120}\left[-3\left(k^{2}\right)_{x} \delta_{x x} u+\left(k^{2}\right)_{x}\left(\delta_{y y} u+\delta_{z z} u\right)\right. \\
& \left.+2\left(k^{2}\right)_{x y} \delta_{y} u+2\left(k^{2}\right)_{x z} \delta_{z} u+\left(\left(k^{2}\right)_{x y y}+\left(k^{2}\right)_{x z z}+k^{2}\left(k^{2}\right)_{x}-\left(k^{2}\right)_{x x x}\right) u\right] \\
= & g-\frac{h^{2}}{6}\left(g_{y y}+g_{z z}+k^{2} g-F_{x}\right)+\frac{h^{4}}{120}\left[g_{y y y y}+2 g_{y y z z}+g_{z z z z}+2 k^{2}\left(g_{y y}+g_{z z}\right)\right. \\
& +2\left(k^{2}\right)_{y} g_{y}+2\left(k^{2}\right)_{z} g_{z}+\left(\left(k^{2}\right)_{y y}+\left(k^{2}\right)_{z z}-3\left(k^{2}\right)_{x x}+k^{4}\right) g \\
& \left.+F_{x x x}-F_{x y y}-F_{x z z}-k^{2} F_{x}\right]+O\left(h^{6}\right) .
\end{aligned}
$$

We now assume that $k$ is constant and the forcing function $F$ is zero in the vicinity of the farfield boundary. Then (5) reduces to

$$
\begin{aligned}
\delta_{x} u= & g-\frac{h^{2}}{6}\left(g_{y y}+g_{z z}+k^{2} g\right) \\
& +\frac{h^{4}}{120}\left[g_{y y y y}+2 g_{y y z z}+g_{z z z z}+2 k^{2}\left(g_{y y}+g_{z z}\right)+k^{4} g\right]+O\left(h^{6}\right) .
\end{aligned}
$$

We have from (2) that $g(y, z)=i \beta u$ and so

$$
\begin{aligned}
\delta_{x} u= & i \beta u-\frac{i \beta h^{2}}{6}\left(u_{y y}+u_{z z}+k^{2} u\right) \\
& +\frac{i \beta h^{4}}{120}\left[u_{y y y y}+2 u_{y y z z}+u_{z z z z}+2 k^{2}\left(u_{y y}+u_{z z}\right)+k^{4} u\right]+O\left(h^{6}\right) .
\end{aligned}
$$

Differentiating (1) twice with respect to $y$ and also with respect to $z$ and adding we get

$$
u_{x x y y}+u_{x x z z}+u_{y y y y}+2 u_{y y z z}+u_{z z z z}+k^{2}\left(u_{y y}+u_{z z}\right)=0 .
$$

Substituting this and (1) into (6) we get

$$
\begin{aligned}
\delta_{x} u & =i \beta u+\frac{i \beta h^{2}}{6} u_{x x}-\frac{i \beta h^{4}}{120}\left[u_{x x y y}+u_{x x z z}-k^{2}\left(u_{y y}+u_{z z}+k^{2} u\right)\right]+O\left(h^{6}\right) \\
& =i \beta u+\frac{i \beta h^{2}}{6}\left(1-\frac{k^{2} h^{2}}{20}\right) u_{x x}-\frac{i \beta h^{4}}{120}\left(u_{x x y y}+u_{x x z z}\right)+O\left(h^{6}\right) .
\end{aligned}
$$

To approximate (2) to sixth order accuracy we need to approximate $u_{x x}$ to fourth order accuracy and the $h^{4}$ term to second order accuracy. By Taylor series

$$
\begin{aligned}
u_{x x} & =\delta_{x x} u-\frac{h^{2}}{12} u_{x x x x}+O\left(h^{4}\right) \\
& =\delta_{x x} u+\frac{h^{2}}{12}\left(u_{x x y y}+u_{x x z z}+k^{2} u_{x x}\right)+O\left(h^{4}\right) \\
& =\left(1+\frac{k^{2} h^{2}}{12}\right) \delta_{x x} u+\frac{h^{2}}{12}\left(u_{x x y y}+u_{x x z z}\right)+O\left(h^{4}\right)
\end{aligned}
$$


Replacing the derivatives in (7) by differences yields a sixth order approximation to (2)

$$
\begin{aligned}
\delta_{x} u= & i \beta u+\frac{i \beta h^{2}}{6}\left(1-\frac{k^{2} h^{2}}{20}\right)\left(1+\frac{k^{2} h^{2}}{12}\right) \delta_{x x} u \\
& +\frac{i \beta h^{4}}{180}\left(1-\frac{k^{2} h^{2}}{8}\right)\left(\delta_{x x y y} u+\delta_{x x z z} u\right)+O\left(h^{6}\right)
\end{aligned}
$$

We are currently performing calculations to compare the accuracy of (3) with (8) for general problems where (4) is not the solution but the artificial boundary is meant to bound the domain when there is an outgoing wave. This is in conjunction with constructing additional high order absorbing boundary conditions for Cartesian coordinates, see [3].

\section{REFERENCES}

[1] Y. Erlangga and E. Turkel, Iterative Schemes for High Order Discretizations to the Exterior Helmholtz Equation. ESAIM: M2AN 46 (2012) 647-660.

[2] E. Turkel, D. Gordon, R. Gordon and S. Tsynkov, Compact 2D and 3D Sixth Order Schemes for the Helmholtz Equation with Variable Wave Number. J. Comput. Phys. 232 (2013) 272-287.

[3] D. Gordon, R. Gordon and E. Turkel, High Order Absorbing Boundary Conditions. To appear in J. Comput. Phys. 